\title{
FACTORES DE RIESGO DE VIOLENCIA FILIO-PARENTAL: UNA APROXIMACIÓN CON JUICIO DE EXPERTOS
}

\author{
CHILD-TO-PARENT VIOLENCE RISK \\ FACTORS: AN APPROACH WITH EXPERT \\ JUDGEMENT
}

\author{
ISMAEL LOINAZ ${ }^{1}$, ANTONIO ANDRÉS-PUEYO ${ }^{1}$ Y \\ ROBERTO PEREIRA ${ }^{2}$
}

Cómo referenciar este artículo/How to reference this article:

Loinaz, I., Andrés-Pueyo, A. y Pereira, R. (2017). Factores de riesgo de violencia filio-parental: Una aproximación con juicio de expertos [Child-to-Parent Violence Risk Factors: An Approach with Expert Judgement]. Acción Psicológica, 14(2), 17-32. https://doi.org/10.5944/ap.14.2.20747

\section{Resumen}

La violencia filio-parental (VFP) es un tipo de violencia familiar, caracterizada por agresiones de distinta índole dirigidas hacia progenitores o personas que ocupen su lugar. Este trabajo describe la creación de la primera herramienta de evaluación del riesgo de VFP, desarrollada a través de la revisión bibliográfica, la opinión de expertos y aplicaciones piloto en contexto clínico. Se analizaron 112 respuestas de profesionales sobre la adecuación de 28 factores de riesgo. También se valoró el interés/utilidad/necesidad de la propuesta de una guía para la valoración del riesgo de VFP. Los resultados mostraron un gran acuerdo entre profesionales, así como una valo- ración positiva de la propuesta. Tras la aplicación piloto de la guía en siete casos y la discusión con expertos, se elaboró la versión final compuesta por 24 factores de riesgo, seis de protección y 15 variables incluidas en una sección inicial.

Palabras clave: Violencia filio-parental; Factores de riesgo; Evaluación del riesgo; Opinión profesional.

\begin{abstract}
Child-to-parent violence (CPV), also known as adolescent-to-parent abuse (APA), is a type of family violence characterized by different kinds aggressions directed to-
\end{abstract}

Correspondencia: Ismael Loinaz. Departamento de Psicología Clínica y Psicobiología. Universitat de Barcelona.

Email: ismael.loinaz@gmail.com

ORCID: Ismael Loinaz (http://orcid.org/0000-0002-6307-6518) y Antonio Andrés-Pueyo (http://orcid.org/0000-00022824-2541)

${ }^{1}$ Universitat de Barcelona, España.

${ }^{2}$ Euskarri, Centro de Formación e Intervención en Violencia Filio-Parental, España.

Recibido: 25 julio de 2017.

Aceptado: 28 de octubre de 2017. 
wards parents or people in their place. This paper describes the development of the first CPV risk assessment tool, derived from bibliographic review, expert opinion, and pilot applications in clinical context. Responses of 112 professionals about the adequacy of 28 factors as well as the interest/utility/need of the proposal of a risk assessment tool for CPV were analyzed. Results showed a great agreement among professionals, as well as a positive evaluation of the proposal. After the pilot application of the tool in 7 cases and discussion with experts, the final version was composed of 24 risk factors and 6 protective factors, as well as 15 variables included in an initial section.

Keywords: Child to parent violence; Risk factors; Violence risk assessment; Professional opinion.

\section{Introducción}

La violencia de jóvenes hacia sus progenitores así como el maltrato a ancianos son fenómenos relativamente nuevos y poco estudiados comparados con la violencia de pareja o el abuso sexual. Las agresiones juveniles se han estudiado tradicionalmente en el ámbito de la delincuencia general. Incluso se afirma que el estudio de la violencia de adolescentes hacia sus padres ha estado infrarrepresentado en políticas publicas y estudios criminológicos (Condry y Miles, 2014). Sin embargo, aunque la violencia filio-parental (VFP) en ocasiones comparta factores con la violencia y delincuencia juvenil, responde a patrones y dinámicas diferentes. Una de las definiciones más repetida de VFP es la de Cottrell (2001) que incluye cualquier acto de un hijo que tiene la intención de causar daño físico, psicológico o económico con el fin de obtener el control sobre uno de los padres (p. 3). Pereira (2006, p. 9) amplío esta definición incluyendo "[...] conductas reiteradas de violencia física (agresiones, golpes, empujones, arrojar objetos), verbal (insultos repetidos, amenazas) o no verbal (gestos amenazadores, ruptura de objetos apreciados) dirigida a los padres o a los adultos que ocupan su lugar. Se excluyen los casos aislados, la [violencia] relacionada con el consumo de tóxicos, la psicopatología grave, la deficiencia mental y el parricidio." A lo largo de este ar- tículo se utilizará el término progenitores para hacer alusión también a aquellas personas que puedan ocupar su lugar y los términos "agresor" o "evaluado" serán igualmente válidos para mujeres.

En los últimos años, la atención prestada a la VFP en España ha crecido exponencialmente superando a la existente a nivel internacional. Las cifras de la Fiscalía General del Estado se mantienen estables tras un repunte en los primeros años de registro, que llegó hasta las 5377 denuncias en 2011. El último dato disponible, de 2015, reflejó 4898 casos y en los dos años anteriores la cifra fue similar (4753 en 2014 y 4659 en 2013), con una media de 4600 casos desde 2007 que hay registros. Sin embargo, un gran número de casos no pasan por la denuncia.

La propia Fiscalía de Menores (2013) señala algunas particularidades de la VFP, como que "[...] raramente se asocia con situaciones de exclusión social [...]" (p. 409) o que "[...] frente al resto de delitos, se caracteriza por que los imputados son tanto varones como mujeres, en proporciones que tienden cada vez más a equipararse [...] (p. 413). En la circular de la Fiscalía 1/2010 se señaló que, pese a la exención penal de los agresores menores de 14 años, existe la necesidad de dedicar recursos de protección e informar sobre la disponibilidad de programas específicos extrajudiciales. Además, " $\mathrm{Si}[\ldots]$ se desprendiera que el menor sigue incurso en los factores de riesgo que le llevaron a cometer actos de violencia doméstica, tales circunstancias habrán de ser comunicadas por el Juzgado a la víctima, a los efectos de que la misma pueda adoptar las medidas de autoprotección que estime oportunas." (Fiscalia, 2010, p. 35).

La investigación sobre evaluación del riesgo de violencia pone de manifiesto la necesidad de atender a la heterogeneidad de las muestras y a la especificidad de los factores de riesgo según el tipo de agresor/violencia, desarrollando herramientas para muestras y objetivos concretos bien sean menores o adultos (Conroy, 2012). Pese a ello, en la actualidad no existe ninguna herramienta para evaluar el riesgo de violencia en casos de VFP. 


\section{Factores de riesgo en VFP}

La bibliografía sobre VFP ha descrito una serie de variables problemáticas tanto en los propios agresores como en sus familias. Al igual que en otras formas de violencia, la transmisión intergeneracional ha sido utilizada como factor explicativo (Aroca, Bellver y Alba, 2012; Boxer, Gullan y Mahoney, 2009), siendo más prevalente la VFP en hijos agredidos por su progenitories (Contreras y Cano, 2016; Izaguirre y Calvete, 2017; Margolin y Baucom, 2014). La bidireccionalidad (que el agresor también sea víctima) es especialmente frecuente en los agresores varones (Ibabe y Jaureguizar, 2011). Por otro lado, la violencia entre los padres se ha relacionado con una mayor frecuencia de violencia del joven hacia los padres (Boxer et al., 2009; Gámez-Guadix y Calvete, 2012). La victimización directa o indirecta podría llegar a explicar entre el $16 \%$ y el $45 \%$ de las agresiones (Rodríguez, González-Álvarez y García-Vera, 2011). También parece existir una relación entre ser víctima de bullying y agredir a compañeros o fuera del colegio. Una explicación provendría de la teoría propuesta por Emler, que vincula la desprotección percibida por la víctima en la situación de intimidación con la búsqueda de una reputación antisocial como forma de autodefensa (Estévez, Inglés, Emler, Martínez-Monteagudo y Torregrosa, 2012). Los agresores podrían utilizar la VFP como medio para compensar los sentimientos de impotencia y expresar su ira en un contexto seguro, lo que Cottrell y Monk (2004) denominan "desplazamiento".

La presencia de otro tipo de agresiones (a compañeros o familiares distintos a los progenitores) ha sido descrita en muestras judiciales como otra de sus principales características). Cuervo, Fernández y Rechea (2008) encontraron que un $65.8 \%$ de los menores juzgados por delitos de VFP también eran violentos fuera del hogar. Pese a analizarse con frecuencia muestras judiciales, muchos casos no llegan a ser una cifra oficial. La VFP aún tiende a mantenerse en secreto, muchas veces por vergüenza (Ghanizadeh y Jafari, 2010; Kennedy, Edmonds, Dann y Burnett, 2010) y los padres se resisten a denunciar hasta que la situación es insostenible (agresiones graves, pérdida absoluta del control, etc.). La existencia de denuncia, por tanto, puede considerarse un indicador de la gra- vedad. La violencia, además, se produce generalmente en escalada y la progresión no se detiene ni siquiera cuando se consigue una sumisión absoluta de la víctima (Pereira y Bertino, 2010).

Un conjunto importante de variables de riesgo hace referencia a las características del agresor. La sintomatología psicopatológica es frecuente en jóvenes que agreden a sus padres, más que en otros jóvenes delincuentes (Kennedy et al., 2010), pudiendo alcanzar una prevalencia del $45 \%$ (Cuervo et al., 2008). Se han descrito de forma habitual el Trastorno por Déficit de Atención con Hiperactividad -TDAH- (Cuervo et al., 2008;), la sintomatología depresiva (Calvete, Orue y Gámez-Guadix, 2013; Castañeda, Garrido-Fernández y Lanzarote, 2012; Ibabe, Arnoso y Elgorriaga, 2014a, 2014b) y los problemas relacionados con el consumo de alcohol y otras drogas (Calvete, Orue y Gámez-Guadix, 2015; Calvete, Orue y Sampedro, 2011; González-Álvarez, Morán y García-Vera, 2011; Ibabe y Jaureguizar, 2011). Modelos de predicción vinculan la conducta antisocial en general, con el consumo problemático de alcohol y la poca supervisión parental (Guillén, Roth, Alfaro y Fernández, 2015). Comparados con otros menores infractores, los casos de VFP presentarían una menor empatía (Ibabe y Jaureguizar, 2011) y una baja autoestima (Cuervo et al., 2008; González-Álvarez et al., 2011; Ibabe y Jaureguizar, 2011; Pereira y Bertino, 2010). Otro rasgo característico sería la baja tolerancia a la frustración con dificultades para afrontar incluso situaciones cotidianas (Cuervo et al., 2008; Cuervo y Rechea, 2010). La impulsividad también es habitual (Calvete et al., 2011; Cuervo et al., 2008; Rico, Rosado y Cantón-Cortés, 2017), llegando hasta el $80 \%$ la prevalencia de esta característica según el criterio de los profesionales (Ibabe, Jaureguizar y Díaz, 2007) y siendo significativamente superiores las puntuaciones respecto a pares no agresores (Castañeda et al., 2012). Asimismo, se encuentra asociada la dificultad para el control de la ira (Cuervo y Rechea, 2010; GonzálezÁlvarez et al., 2011). Por último, dentro de estos rasgos de la personalidad, el narcisismo o las ideas de grandiosidad también se han vinculado a la VFP (Calvete, 2008; Calvete et al., 2011).

En relación a los procesos de adaptación del agresor, lo más repetido son las dificultades académicas (Cuervo 
et al., 2008; González-Álvarez, Gesteira, FernándezArias y García-Vera, 2010; González-Álvarez et al., 2011). Los problemas de adaptación, aprendizaje, absentismo y/o cambio de centro, junto a la agresión a compañeros, son más frecuentes en estos jóvenes que en sus pares no violentos (Castañeda et al., 2012). Por otro lado, la conducta antisocial se ha relacionado con un número mayor de variables de mal pronóstico comparado con casos limitados a la VFP (Ibabe et al., 2007). La propensión a la delincuencia también sería significativamente mayor en jóvenes que agreden a sus padres comparados con pares no agresores (Castañeda et al., 2012). Las relaciones antisociales también tienen un fuerte impacto en el desarrollo de la violencia (Hong, Kral, Espelage y AllenMeares, 2012), fomentando su uso para ganar poder y control (Cottrell y Monk, 2004), en especial si la supervisión familiar está ausente (Cutrín, Gómez-Fraguela y Luengo, 2015).

De especial interés son los factores familiares. La variable repetida en la mayoría de casos es el estilo educativo problemático (Aroca, Miró y Bellver, 2013; Castañeda et al., 2012; Cuervo et al., 2008; Cuervo y Rechea, 2010). En general, son habituales la baja disciplina (Calvete et al., 2011), la permisividad y la negligencia (Aroca, Cánovas y Alba, 2012). Este último estilo incrementa la probabilidad de violencia física y verbal (Contreras y Cano, 2014; Gamez-Guadix, Jaureguizar, Almendros y Carrobles, 2012). Vinculada al estilo educativo se encuentra la inversión de la jerarquía, consistente en que el agresor suplanta el rol de autoridad de los progenitores, dificultando el establecimiento de normas y límites (Pérez y Pereira, 2006), pudiendo llegar a la dominación y trato del progenitor como sirviente (Stewart, Burns y Leonard, 2007). Es frecuente que los padres, en estas situaciones, traten de delegar en un tercero el establecimiento de normas (Pereira y Bertino, 2010). Este "abandono" del rol paterno en ocasiones se describe como un mecanismo de protección (Tew y Nixon, 2010).

En estas familias, además, los problemas de convivencia distintos a la propia VFP son frecuentes, con dinámicas que contribuyen al desarrollo de conductas violentas (Ibabe et al., 2007). Los ciclos coercitivos mutuos y los mensajes inapropiados de tipo acusatorio dan pie al uso de la violencia como estrategia disuasoria (Pagani et al., 2004, 2009). La presencia de conflictos no violentos entre los progenitores (de poder, estilos de comunicación inadecuados, etc.) también supone otro factor de riesgo (Ibabe et al., 2007) que se puede traducir en una incapacidad para establecer normas coherentes (Pereira y Bertino, 2010). Además, hay problemas propios de los progenitores, como las adicciones y otros trastornos psicológicos, que se han relacionado con la VFP (Cuervo et al. 2008). El consumo de drogas de las madres, por ejemplo, incrementaría el riesgo de VFP en mayor medida que el consumo problemático por parte del agresor (Pagani et al., 2004), aunque no ocurre lo mismo con el consumo del padre (Pagani et al., 2009).

Junto a los factores de riesgo, la bibliografía describe factores de protección que tienden a ser comunes en distintos tipos de violencia y formas delictivas. Incluso desarrollos recientes proponen herramientas cuyos factores pueden ser considerados de riesgo y/o de protección según el caso o el contexto (ver Loinaz, 2017). Estos factores no se han descrito de forma específica en la VFP, aunque en muchos casos la ausencia de los problemas mencionados en los párrafos anteriores puede considerarse protector. En los casos de VFP es necesario intervenir en el circuito en el que se establece la violencia (Aroca, Bellver, et al., 2012; Pereira y Bertino, 2010). Por ello, la vinculación familiar en la terapia incrementa la consecución de los objetivos del tratamiento, siendo un factor protector en menores infractores (Contreras, Molina y Cano, 2011). Otros factores de protección genéricos que pueden resultar relevantes en menores infractores son la existencia de planes de futuro prosociales (sirven como meta hacia la que dirigir los objetivos), el soporte social (presencia de amigos y referentes adultos tutores, monitores, educadores-) y el soporte familiar (no solo para la persona agresora sino también para la víctima).

El objetivo de esta investigación fue proponer una guía para la evaluación del riesgo de VFP que cubriera todas las particularidades y necesidades mencionadas en esta introducción. Además, se espera crear una herramienta con consenso y aval de los profesionales a los que va dirigida y adaptada al uso diario en los contextos en los que se trabaja con estos casos, algo fundamental para la buena utilización de la misma. 


\section{Método}

\section{Participantes}

Un total de 160 profesionales participaron en la encuesta, de los que se analizaron 112 respuestas completas. Los participantes se dedicaban a la psicología (39.3\%), educación social $(24.1 \%)$, policía $(9.8 \%)$, trabajo social $(6.3 \%)$ y docencia/investigación (5.4\%) entre otras profesiones. La edad media fue de 41 años $(D T=9.9 ;$ rango $=23-66)$ y el $46.4 \%$ fueron mujeres. El $58 \%$ tenía formación en evaluación del riesgo, el $67.6 \%$ de ellos académica (estudios como criminología, psicología forense) y el $80 \%$ en talleres específicos. El $79.5 \%$ estaba trabajando directamente en casos de VFP. De los que en la actualidad no trabajaban en el ámbito, un $43.4 \%$ lo había hecho con anterioridad.

\section{Material}

A partir de la revisión bibliográfica, se elaboró una encuesta que contenía 28 factores (Tabla 1), agrupados en cuatro dimensiones: 1) características de la violencia; 2) características del agresor; 3) características familiares; y 4) factores protectores. Se consultó la adecuación de estos factores para evaluar casos de VFP (adecuado, ns/nc, inadecuado), así como la opinión (Tabla 2) sobre la creación de una herramienta para valorar el riesgo en estos casos, permitiendo, además, aportar cualquier sugerencia u opinión que considerasen oportuna para la construcción del protocolo de valoración del riesgo.

\section{Procedimiento}

Distintos profesionales fueron contactados vía email para solicitar su colaboración. Dicho contacto incluyó ámbitos académicos, centros de atención y tratamiento a menores, servicios relacionados con la justicia juvenil y cuerpos policiales. Además, se difundió en las webs de la Sociedad Española para el Estudio de la Violencia FilioParental (SEVIFIP), de Euskarri (Centro de Formación e Intervención en Violencia Filio-Parental; Bilbao) y del grupo de investigación Deusto Stress Research. La participación tuvo lugar entre abril y junio de 2014.
Tras analizar los resultados de la encuesta y elaborar la primera versión de la herramienta, se realizaron siete aplicaciones piloto en el centro Euskarri en junio de 2014. El objetivo de esta fase fue poner a prueba el protocolo, la descripción de los ítems y su facilidad de uso. Además, los expertos informaron sobre cuestiones relacionadas con la aplicabilidad de la herramienta. En diciembre de 2014 también se realizó una sesión clínica con un panel de expertos en el centro Amalgama 7 (Barcelona).

\section{Resultados}

\section{Adecuación de los factores}

La opinión de los profesionales respecto a la adecuación o no de los factores de riesgo para evaluar casos de VFP se presenta en la Tabla 1. El grupo de variables consideradas más adecuadas (porcentaje superior al $90 \%)$ fue el correspondiente a cuestiones familiares (violencia entre los padres, problemas de convivencia, estilo educativo, soporte familiar y vinculación familiar en la terapia). Respecto al propio agresor, las variables consideradas más adecuadas fueron el estilo afectivo, el abuso de sustancias y la dificultad para el control de la ira. Los factores que obtuvieron un menor apoyo profesional fueron el narcisismo o ideas de grandiosidad $(67.3 \%)$, los conflictos no violentos entre los padres $(66.4 \%)$, la monoparentalidad $(62.8 \%)$ y la familia adoptiva $(64.6 \%)$.

\section{Opinión sobre la propuesta de herra- mienta}

Entre el total de los encuestados, se consideró la propuesta bastante $(45.65 \%)$ o muy $(38.04 \%)$ necesaria; que cubría bastante $(70.65 \%)$ o perfectamente $(10.87 \%)$ sus necesidades profesionales; bastante $(54.35 \%)$ o muy útil (14.13\%) para su labor en casos de VFP; cubría las áreas de interés en VFP (88\%); y que podía ser sencilla 
Tabla 1

Consideración de los factores como adecuados o inadecuados para evaluar el riesgo en casos de VFP $(n=112)$

\begin{tabular}{|c|c|c|c|}
\hline \multicolumn{2}{|c|}{ Factor } & \multirow{2}{*}{$\frac{\text { Adecuado \% }}{87.6}$} & \multirow{2}{*}{$\frac{\text { Inadecuado } \%}{8.8}$} \\
\hline 1. & Ser víctima de violencia en el hogar. & & \\
\hline 2. & Ser víctima de bullying. & 75.2 & 10.0 \\
\hline 3. & Ejercer violencia fuera del hogar. & 79.6 & 8.8 \\
\hline 4. & Existe denuncia por VFP. & 86.7 & 7.1 \\
\hline 5. & Existe escalada en la VFP. & 82.3 & 6.2 \\
\hline 6. & $\begin{array}{l}\text { Trastorno psicológico: TDAH, depresión, trastorno negativista desafiante o } \\
\text { rasgos antisociales. }\end{array}$ & 81.4 & 11.5 \\
\hline 7. & Estilo afectivo: baja autoestima, poca capacidad empática. & 90.3 & 5.3 \\
\hline 8. & Intolerancia a la frustración. & 87.6 & 3.5 \\
\hline 9. & Abuso de sustancias. & 91.2 & 2.7 \\
\hline 10. & Narcisismo o ideas de grandiosidad. & 67.3 & 19.5 \\
\hline 11. & Impulsividad. & 88.5 & 3.5 \\
\hline 12. & Dificultad para el control de la ira. & 96.5 & 0.9 \\
\hline 13. & Dificultades académicas. & 71.7 & 14.2 \\
\hline 14. & Conducta delictiva al margen de la VFP. & 80.5 & 13.3 \\
\hline 15. & Pares y amistades delictivas, antisociales. & 82.3 & 9.7 \\
\hline 16. & Violencia entre los padres. & 92.9 & 3.5 \\
\hline 17. & Conflictos no violentos entre los padres. & 66.4 & 16.8 \\
\hline 18. & Problemas de convivencia: luchas de poder, comunicación deficiente. & 92.9 & 0.9 \\
\hline 19. & Estilo educativo: permisivo, negligente, rechazante o autoritario. & 97.3 & 2.7 \\
\hline 20. & Inversión de la jerarquía. & 87.6 & 4.4 \\
\hline 21. & $\begin{array}{l}\text { Monoparentalidad: si se ha reducido el apoyo del entorno familiar, causa } \\
\text { problemas económicos. }\end{array}$ & 62.8 & 18.6 \\
\hline 22. & Problemas en los progenitores: psicopatológicos o de adicción. & 77.9 & 8.0 \\
\hline 23. & Familia adoptiva. & 64.6 & 15.0 \\
\hline 24. & Madre principal objetivo de la violencia. & 86.7 & 7.1 \\
\hline 25. & Planes de futuro: académicos, laborales. & 83.2 & 4.5 \\
\hline 26. & Soporte social: amistades, red social. & 85.8 & 5.3 \\
\hline 27. & Soporte familiar: apoyo de familiares para favorecer su desarrollo positivo. & 97.3 & 1.8 \\
\hline 28. & Vinculación familiar en la terapia. & 93.8 & 1.8 \\
\hline
\end{tabular}

de aplicar $(89.13 \%)$. Respecto a la utilidad, la consideraron: bastante $(48.91 \%)$ o muy útil $(6.52 \%)$ para valorar la posible evolución del caso; bastante $(65.2 \%)$ o muy $(20.65 \%)$ útil para valorar las necesidades de intervención; bastante (56.04 \%) o muy (14.29\%) útil para recomendar un tratamiento; bastante $(37.36 \%$ ) o muy $(10.99 \%)$ útil para predecir la reincidencia; y bastante $(47.83 \%)$ o muy (17.39\%) útil para valorar el riesgo que corren los familiares.

En la Tabla 2 se comparan estas opiniones entre aquellos que trabajaban o no en VFP y entre los que tenían o no formación en evaluación del riesgo. Las diferencias fueron significativas solo en que los que no trabajaban en VFP consideraron la propuesta más necesaria y más útil para predecir la reincidencia, y en que los no formados en evaluación del riesgo la consideraron más útil para valorar el riesgo en familiares.

\section{Propuestas de los encuestados}

Los participantes hicieron distintas propuestas de mejora entre las que se encontraba la propuesta de nuevos factores de riesgo que ellos consideraban relevantes y no estaban presentes. En total se incluyeron 9 factores nuevos: edad de inicio de la VFP; comisión de VFP por parte 
Tabla 2

Opinión sobre la propuesta de herramienta

\begin{tabular}{|c|c|c|c|c|c|c|c|}
\hline & & \multicolumn{3}{|c|}{ Trabajan en VFP } & \multicolumn{3}{|c|}{$\begin{array}{l}\text { Formados en evaluación del } \\
\text { riesgo }\end{array}$} \\
\hline & & $\begin{array}{c}\text { Sí } \\
(n=89)\end{array}$ & $\begin{array}{c}\text { NO } \\
(n=23)\end{array}$ & & $\begin{array}{c}\text { Sí } \\
(n=65)\end{array}$ & $\begin{array}{c}\text { NO } \\
(n=47)\end{array}$ & \\
\hline \multirow{4}{*}{$\begin{array}{l}\text { Cubre necesidades } \\
\text { contexto de trabajo }\end{array}$} & & $\%(n)$ & $\%(n)$ & $p$ & $\%(n)$ & $\%(n)$ & $p$ \\
\hline & Perfectamente & $9 \%(8)$ & $13 \%(3)$ & \multirow{3}{*}{659} & $4.6 \%(3)$ & $17 \%(8)$ & \multirow{3}{*}{.085} \\
\hline & Bastante & $70.8 \%(63)$ & $73.9 \%(17)$ & & $76.9 \%(50)$ & $63.8 \%(30)$ & \\
\hline & Algo & $20.2 \%(18)$ & $13 \%(3)$ & & $18.5 \%(12)$ & $19.1 \%(9)$ & \\
\hline \multirow{4}{*}{ Útil } & Muy & $12.4 \%(11)$ & $26.1 \%(6)$ & \multirow{4}{*}{.197} & $12.3 \%(8)$ & $19.1 \%(9)$ & \multirow{4}{*}{.445} \\
\hline & Bastante & $52.8 \%(47)$ & $56.5 \%(13)$ & & $50.8 \%(33)$ & $57.4 \%(27)$ & \\
\hline & Algo & $28.1 \%(25)$ & $17.4 \%(4)$ & & $30.8 \%(20)$ & $19.1 \%(9)$ & \\
\hline & Poco & $6.7 \%(6)$ & $0 \%(0)$ & & $6.2 \%(4)$ & $4.3 \%(2)$ & \\
\hline \multirow{4}{*}{ Necesaria } & Muy & $31.5 \%(28)$ & $69.6 \%(16)$ & \multirow{4}{*}{.009} & $38.5 \%(25)$ & $40.4 \%(19)$ & \multirow{4}{*}{.783} \\
\hline & Bastante & $51.7 \%(46)$ & $26.1 \%(6)$ & & $46.2 \%(30)$ & $46.8 \%(22)$ & \\
\hline & Algo & $11.2 \%(10)$ & $4.3 \%(1)$ & & $9.2 \%(6)$ & $10.6 \%(5)$ & \\
\hline & Poco & $5.6 \%(5)$ & $0 \%(0)$ & & $6.2 \%(4)$ & $2.1 \%(1)$ & \\
\hline \multirow{2}{*}{$\begin{array}{l}\text { Ítems cubren áreas de } \\
\text { interés }\end{array}$} & Sí & $89.9 \%(80)$ & $91.3 \%(21)$ & \multirow{2}{*}{.839} & $86.2 \%(56)$ & $95.7 \%(45)$ & \multirow{2}{*}{.092} \\
\hline & No & $10.1 \%(9)$ & $8.7 \%(2)$ & & $13.8 \%(9)$ & $4.3 \%(2)$ & \\
\hline \multirow[t]{2}{*}{ Aplicación sencilla } & Sí & $88.8 \%(79)$ & $100 \%(23)$ & \multirow{2}{*}{.092} & $89.2 \%(58)$ & $93.6 \%(44)$ & \multirow{2}{*}{.422} \\
\hline & No & $11.2 \%(10)$ & $0 \%(0)$ & & $10.8 \%(7)$ & $6.4 \%(3)$ & \\
\hline \multicolumn{8}{|l|}{ Útil para pronosticar... } \\
\hline \multirow{5}{*}{ Evolución del caso } & Nada & $2.2 \%(2)$ & $0 \%(0)$ & \multirow{5}{*}{.268} & $1.5 \%(1)$ & $2.1 \%(1)$ & \multirow{5}{*}{.100} \\
\hline & Poco & $12.4 \%(11)$ & $13 \%(3)$ & & $16.9 \%(11)$ & $6.4 \%(3)$ & \\
\hline & Algo & $37.1 \%(33)$ & $21.7 \%(5)$ & & $40 \%(26)$ & $25.5 \%(12)$ & \\
\hline & Bastante & $44.9 \%(40)$ & $52.2 \%(12)$ & & $38.5 \%(25)$ & $57.4 \%(27)$ & \\
\hline & Mucho & $3.4 \%(3)$ & $13 \%(3)$ & & $3.1 \%(2)$ & $8.5 \%(4)$ & \\
\hline \multirow{4}{*}{$\begin{array}{l}\text { Necesidades de } \\
\text { intervención }\end{array}$} & Poco & $5.6 \%(5)$ & $0 \%(0)$ & \multirow{4}{*}{.428} & $6.2 \%(4)$ & $2.1 \%(1)$ & \multirow{4}{*}{.168} \\
\hline & Algo & $9 \%(8)$ & $17.4 \%(4)$ & & $15.4 \%(10)$ & $4.3 \%(2)$ & \\
\hline & Bastante & $67.4 \%(60)$ & $69.6 \%(16)$ & & $61.5 \%(40)$ & $76.6 \%(36)$ & \\
\hline & Mucho & $18 \%(16)$ & $13 \%(3)$ & & $16.9 \%(11)$ & $17 \%(8)$ & \\
\hline & Poco & $10.1 \%(9)$ & $8.7 \%(2)$ & & $12.3 \%(8)$ & $6.4 \%(3)$ & \\
\hline & Algo & $19.1 \%(17)$ & $26.1 \%(6)$ & & $20 \%(13)$ & $21.3 \%(10)$ & \\
\hline Recomendar tratamiento & Bastante & $60.7 \%(54)$ & $47,8 \%(11)$ & .609 & $60 \%(39)$ & $55.3 \%(26)$ & .370 \\
\hline & Mucho & $10.1 \%(9)$ & $17.4 \%(4)$ & & $7.7 \%(5)$ & $17 \%(8)$ & \\
\hline & Nada & $5.6 \%(5)$ & $0 \%(0)$ & & $6.2 \%(4)$ & $2.1 \%(1)$ & \\
\hline & Poco & $14.6 \%(13)$ & $13 \%(3)$ & & $15.4 \%(10)$ & $12.8 \%(6)$ & \\
\hline Reincidencia & Algo & $34.8 \%(31)$ & $39.1 \%(9)$ & .032 & $38.5 \%(25)$ & $31.9 \%(15)$ & .574 \\
\hline & Bastante & $39.35(35)$ & $4.3 \%(1)$ & & $30.8 \%(20)$ & $44.7 \%(21)$ & \\
\hline & Mucho & $5.6 \%(5)$ & $21.7 \%(5)$ & & $9.2 \%(6)$ & $8.5 \%(4)$ & \\
\hline & Nada & $3,4 \%(3)$ & $0 \%(0)$ & & $4.6 \%(3)$ & $0 \%(0)$ & \\
\hline & Poco & $10.1 \%(9)$ & $4.3 \%(1)$ & & $12.3 \%(8)$ & $4.3 \%(2)$ & \\
\hline Riesgo familiares & Algo & $23.6 \%(21)$ & $30.4 \%(7)$ & .267 & $30.8 \%(20)$ & $17 \%(8)$ & .044 \\
\hline & Bastante & $51.7 \%(46)$ & $39.1 \%(9)$ & & $38.5 \%(25)$ & $63.8 \%(30)$ & \\
\hline & Mucho & $11.2 \%(10)$ & $26.1 \%(6)$ & & $13.8 \%(9)$ & $14.9 \%(7)$ & \\
\hline
\end{tabular}

de hermanos/as; actitudes frente al uso de la violencia; gen; alianza terapéutica; víctimas de violencia de género falta de valores; tipo de violencia ejercida; fallo en intervenciones anteriores; migración, reagrupación familiar, separación temporal entre padres e hijos, cultura de ori(madre o hija agresora). También se propuso la utilización de un lenguaje inclusivo que dejara clara la po- 
sibilidad de que ambos sexos pudieran ser víctimas y agresores.

Tras las aplicaciones piloto y los grupos de discusión, se incluyeron distintas modificaciones que dieron lugar a la versión 2 de la guía. Los ítems que contaban con más apoyo empírico se mantuvieron como factores principales. Los demás (e.g., monoparentalidad, familia adoptiva y madre víctima), pasaron a formar parte de las variables identificativas del caso junto a variables sociodemográficas. El factor de riesgo 7 (ver Tabla 1) se dividió en dos (7. problemas de empatía y 8. problemas de autoestima). Se incorporaron como nuevos factores las actitudes violentas, el fallo en intervenciones previas, la motivación para el cambio y la alianza terapéutica. La versión final de la herramienta (v2.0; disponible bajo petición a los autores) contiene un total de 24 factores de riesgo, 6 de protección y 15 variables identificativas del caso.

\section{Discusión}

La revisión bibliográfica pone de manifiesto una serie de variables que se repiten en la investigación sobre VFP y que pueden considerarse factores de riesgo. Estas variables fueron sometidas en 2014 a la consideración de profesionales de la materia para ser incorporadas a una herramienta para la valoración del riesgo de violencia. Muchos de los factores habían sido revisados con anterioridad (Ibabe, Jaureguizar y Bentler, 2013; Lozano, Estévez y Carballo, 2013) y lo han sido con posterioridad al estudio (Martínez, Estévez, Jiménez y Velilla, 2015). Otros, sin embargo, son menos habituales. La investigación también ha puesto de manifiesto la necesidad de consensuar definiciones y desarrollar herramientas para la valoración del riesgo de violencia en casos de VFP (Holt, 2013), así como entender cómo determinadas variables se convierten en factores de riesgo en poblaciones concretas (Holt, 2012).

El presente trabajo sirvió para elaborar una herramienta consensuada con los profesionales que en su práctica diaria trabajan con casos de VFP y avalada, además, por la bibliografía. El hecho de tener en cuenta la opinión de los destinatarios de la herramienta ha sido puesto de manifiesto desde los orígenes de la predicción delictiva (Tibbitts, 1932) y forma parte del procedimiento de crea- ción y revisión de las principales herramientas de juicio estructurado (ver Douglas et al., 2014). Respecto a la herramienta, la principal conclusión es que los profesionales la consideraron necesaria y útil. Los factores de riesgo recibieron soporte y además se obtuvo la propuesta de nueve factores poco estudiados hasta la fecha (e.g., monoparentalidad, familia adoptiva, fallecimiento de algún progenitor, hermanos agresores, edad de comienzo de la VFP). La aplicación piloto de la versión inicial permitió solventar los principales problemas que surgían en su uso en la práctica, tras lo cual se dispuso de una herramienta que en la actualidad está siendo aplicada de forma experimental en distintos contextos.

El trabajo aún supone una primera fase en el desarrollo de una herramienta para la evaluación del riesgo de VFP. Las siguientes etapas deberán establecer su contenido definitivo y sus propiedades (e.g., capacidad predictiva, validez convergente, fiabilidad interjueces, etc.), incluyendo la demostración del aporte diferencial que supone respecto a otras herramientas disponibles, como el SAVRY (Borum, Bartel y Froth, 2006), por ejemplo, para muestras juveniles (aunque debemos recordar que los implicados en VFP no necesariamente deben ser menores de edad). Asimismo, se deberá poner a prueba su utilidad en distintos contextos, como puede ser la valoración del progreso en intervenciones clínicas, el ajuste de medidas judiciales o la respuesta policial frente a denuncias por VFP que recientemente está cobrando especial interés (Miles y Condry, 2016).

\section{Referencias}

Aroca, C., Bellver, M. C. y Alba, J. L. (2012). La teoría del aprendizaje social como modelo explicativo de la violencia filio-parental [The Social Learning Theory as Explicative Model of Child-Parent Violence]. Revista Complutense de Educación, 23, 487-511.

https://doi.org/10.5209/rev_RCED.2012.v23.n2.400 39 
Aroca, C., Cánovas, P. y Alba, J. L. (2012). Características de las familias que sufren violencia filio-parental: un estudio de revisión [Characteristics of Families Suffering Parent Abuse: A Literature Review]. Educatio Siglo XXI, 30, 231-254. Recuperado de http://revistas.um.es/educatio/article/viewFile/16080 $1 / 140811$

Aroca, C., Miró, C. y Bellver, M. C. (2013). Los problemas de violencia en los adolescentes. ¿Incapacidad educativa de los progenitores? [The Problems of Violence in Adolescents. Inability of the Parents to Transmit Values?] Educación Social: Revista de Intervención Socioeducativa, 53, 121$136 . \quad$ Recuperado de http://www.raco.cat/index.php/EducacioSocial/articl e/viewFile/263620/368975

Borum, R., Bartel, P. y Forth, A. (2006). Manual for the Structured Assessment for Violence Risk in Youth (SAVRY). Odessa, FL: Psychological Assessment Resources.

Boxer, P., Gullan, R. L. y Mahoney, A. (2009). Adolescents' physical aggression toward parents in a clinic-referred sample. Journal of Clinical Child \& Adolescent Psychology, 38, 106-116. https://doi.org/10.1080/15374410802575396

Calvete, E. (2008). Justification of violence and grandiosity schemas as predictors of antisocial behavior in adolescents. Journal of Abnormal Child Psychology, 36, 1083-1095. https://doi.org/10.1007/s10802-008-9229-5

Calvete, E., Orue, I. y Gámez-Guadix, M. (2013). Childto-parent violence: Emotional and behavioral predictors. Journal of Interpersonal Violence, 28, 755-772. https://doi.org/10.1177/0886260512455869

Calvete, E. , Orue, I. y Gámez-Guadix, M. (2015). Reciprocal longitudinal associations between substance use and child-to-parent violence in adolescents. Journal of Adolescence, 44, 124-
133.

https://doi.org/10.1016/j.adolescence.2015.07.015

Calvete, E., Orue, I. y Sampedro, R. (2011). Violencia filio-parental en la adolescencia: Características ambientales y personales [Child to Parent Violence in Adolescence: Environmental and Individual Characteristics]. Infancia y Aprendizaje, 34, 349363. https://doi.org/10.1174/021037011797238577

Castañeda, A., Garrido-Fernández, M. y Lanzarote, M. D. (2012). Menores con conducta de maltrato hacia los progenitores: Un estudio de personalidad y estilos de socialización [Juvenile Offenders who Assault their Parents: A Study of Personality Traits and Parenting Styles]. Revista de Psicología Social, $27(2)$, 157-167. https://doi.org/10.1174/021347412800337933

Condry, R. y Miles, C. (2014). Adolescent to parent violence: Framing and mapping a hidden problem. Criminology and Criminal Justice, 14, 257-275. https://doi.org/10.1177/1748895813500155

Conroy, M. A. (2012). Assessing juveniles for risk of violence. En E. L. Grigorenko (Ed.), Handbook of Juvenile Forensic Psychology and Psychiatry (pp. 227-238). New York, NY: Springer.

Contreras, L. y Cano, C. (2014). Family profile of young offenders who abuse their parents: A comparison with general offenders and non-offenders. Journal of Family Violence, 29, 901-910. https://doi.org/10.1007/s10896-014-9637-y

Contreras, L. y Cano, M. C. (2016). Child-to-parent violence: the role of exposure to violence and its relationship to social-cognitive processing. European Journal of Psychology Applied to Legal Context, $8(2), \quad 43-50$. https://doi.org/10.1016/j.ejpal.2016.03.003

Contreras, L., Molina, V. y Cano, M. C. (2011). In search of psychosocial variables linked to the recidivism in young offenders. European Journal of Psychology Applied to Legal Context, 3, 77-88. 
Cottrell, B. (2001). Parent Abuse: The Abuse of Parents by their Teenage Children. Ottawa, ON: Health Canada, Population and Public Health Branch, National Clearinghouse on Family Violence. Recuperado de http://publications.gc.ca/collections/Collection/H7 2-21-180-2000E.pdf

Cottrell, B. y Monk, P. (2004). Adolescent-to-parent abuse - A qualitative overview of common themes. Journal of Family Issues, 25, 1072-1095. https://doi.org/10.1177/0192513x03261330

Cuervo, A. L., Fernández, E. y Rechea, C. (2008). Menores agresores en el hogar [Minor Offenders in the Home]. Boletín Criminológico, 106, 1-4.

Cuervo, A. L. y Rechea, C. (2010). Menores agresores en el ámbito familiar. Un estudio de casos [Minor Offenders in the Family. A Case Study]. Revista de Derecho Penal y Criminología, 33), 353-375. Recuperado de http://espacio.uned.es/fez/eserv.php?pid=bibliuned:revista DerechoPenalyCriminologia-2010-3-

5090\&dsID=Documento.pdf

Cutrín, O., Gómez-Fraguela, J. A. y Luengo, M. Á. (2015). Peer-group mediation in the relationship between family and juvenile antisocial behavior. European Journal of Psychology Applied to Legal Context, 7, 59-65. https://doi.org/10.1016/j.ejpal.2014.11.005

Douglas, K. S., Hart, S. D., Webster, C. D., Belfrage, H., Guy, L. S. y Wilson, C. M. (2014). HistoricalClinical-Risk Management-20, version 3 (HCR20V3): Development and overview. International Journal of Forensic Mental Health, 13, 93-108. https://doi.org/10.1080/14999013.2014.906519

Estévez, E., Inglés, C. J., Emler, N. P., MartínezMonteagudo, M. C. y Torregrosa, M. S. (2012). Análisis de la relación entre la victimización y la violencia escolar: El rol de la reputación antisocial. Psychosocial Intervention, 21, 53-65. https://doi.org/10.5093/in2012v21n1a3
Fiscalía (2010). Circular 1/2010, de 23 de julio, Sobre el tratamiento desde el sistema de justicia juvenil de los malos tratos de los menores contra sus ascendientes [On the Treatment of the Juvenile Justice System of Maltraitment against its Ascendants. Recuperado de http://jurisprudencia.vlex.es/vid/-230985942

Fiscalía (2013). Memoria de la Fiscalía 2012 (Apartado de la Sala de Coordinadora en materia de Menores) [Report of the Prosecutor's Office 2012](Section of the Coordinating Board on Minors) . Recuperado de https://www.fiscal.es

Gámez-Guadix, M. y Calvete, E. (2012). Violencia filioparental y su asociación con la exposición a la violencia marital y la agresión de padres a hijos [Child-to-Parent Violence and its Association with Exposure to Marital Violence and Parent-to-Child Violence]. Psicothema, 24, 277-283. Recuperado de http://www.psicothema.com/psicothema.asp?id=401 1

Gamez-Guadix, M., Jaureguizar, J., Almendros, C. y Carrobles, J. A. (2012). Estilos de socialización familiar y violencia de hijos a padres en población española [Parenting Styles and Child to Parent Violence in Spanish Population]. Behavioral Psychology-Psicología Conductual, 20, 585-602.

Ghanizadeh, A. y Jafari, P. (2010). Risk factors of abuse of parents by their ADHD children. European Child \& Adolescent Psychiatry, 19, 75-81.

González-Álvarez, M., Gesteira, C., Fernández-Arias, I. y García-Vera, M. P. (2010). Adolescentes que agreden a sus padres. Un análisis descriptivo de los menores agresores [Teens Assaulting their Parents. A Descriptive Analysis of the Minor Aggressors. Psicopatología Clínica, Legal y Forense, 10, 37-53. Recuperado http://masterforense.com/pdf/2010/2010art3.pdf

González-Álvarez, M., Morán, N. y García-Vera, M. P. (2011). Violencia de hijos a padres: Revisión teórica de las variables clínicas descriptoras de los menores agresores [Violence of Children to Parents: 
Theoretical Review of the Descriptive Clinical Variables of the Minor Aggressors]. Psicopatología Clínica, Legal y Forense, 11, 101-121. Recuperado de http://masterforense.com/pdf/2011/2011art6.pdf

Guillén, N. Roth, E., Alfaro, A. y Fernández, E. (2015). Youth alcohol drinking behavior: Associated risk and protective factors. Revista Iberoamericana de Psicología y Salud, 6, 53-63. https://doi.org/10.1016/j.rips.2015.03.001

Holt, A. (2012). Researching parent abuse: A critical review of the methods. Social Policy and Society, 11 , 289-298. https://doi.org/10.1017/S1474746411000625

Holt, A. (2013). Adolescent-to-Parent Abuse. Bristol, UK: Policy Press.

Hong, J. S., Kral, M. J., Espelage, D. L. y Allen-Meares, P. (2012). The social ecology of adolescent-initiated parent abuse: A review of the literature. Child Psychiatry and Human Development, 43, 431-454. https://doi.org/10.1007/s10578-011-0273-y

Ibabe, I., Arnoso, A. y Elgorriaga, E. (2014a). Behavioral problems and depressive symptomatology as predictors of child-to-parent violence. European Journal of Psychology Applied to Legal Context, 6, 53-61. https://doi.org/10.1016/j.ejpal.2014.06.004

Ibabe, I., Arnoso, A. y Elgorriaga, E. (2014b). The clinical profile of adolescent offenders of child-toparent violence. Procedia - Social and Behavioral Sciences, $\quad 131, \quad 377-381$. https://doi.org/10.1016/j.sbspro.2014.04.133

Ibabe, I. y Jaureguizar, J. (2011). ¿Hasta qué punto la violencia filio-parental es bidireccional? [¿Hasta qué punto la violencia filio-parental es bidireccional?] Anales de Psicología, 27, 265-277. Recuperado de: http://www.redalyc.org/pdf/167/16720051001.pdf

Ibabe, I., Jaureguizar, J. y Bentler, P. M. (2013). Risk factors for child-to-parent violence. Journal of
Family Violence, 28, 523-534. https://doi.org/10.1007/s10896-013-9512-2

Ibabe, I., Jaureguizar, J. y Díaz, O. (2007). Violencia filio-parental: Conductas violentas de jóvenes hacia sus padres. Vitoria-Gasteiz, España: Servicio Central de Publicaciones del Gobierno Vasco. Recuperado de http://www.jusap.ejgv.euskadi.eus/r47edukia/es/contenidos/documentacion/violencia filio parental/es_vifilpar/adjuntos/Violencia_FilioParental.pdf

Izaguirre, A. y Calvete, E. (2017). Exposure to family violence as a predictor of dating violence and childto-parent aggression in Spanish adolescents. Youth \& Society, 49(3), 393-412. https://doi.org/10.1177/0044118X16632138

Kennedy, T. D., Edmonds, W. A., Dann, K. T. J. y Burnett, K. F. (2010). The clinical and adaptive features of young offenders with histories of childparent violence. Journal of Family Violence, 25, 509-520. https://doi.org/10.1007/s10896-010-9312$\mathrm{x}$

Loinaz, I. (2017). Manual de evaluación del riesgo de violencia. Metodología y ámbitos de aplicación [Manual of Evaluation of the Risk of Violence. Methodology and Fields of Application. Madrid, España: Pirámide.

Loinaz, I. y Sánchez, N. (2015). El ciclo victimizaciónagresión y el maltrato infantil [The Cycle of Victimization-Aggression and Child Abuse]. En I. Loinaz y R. Gallardo (Eds.), Prevención y detección del maltrato infantil (pp. 13-49). Puebla, México: Benemérita Universidad Autónoma de México.

Lozano, S., Estévez, E. y Carballo, J. L. (2013). Factores individuales y familiares de riesgo en casos de violencia filio-parental [Individual and Family Risk Factors in Cases of Filio-Parental Violence]. Documentos de Trabajo Social, 52, 239-254. Recuperado 
https://dialnet.unirioja.es/servlet/articulo? $\operatorname{codigo}=47$ 03109

Margolin, G. y Baucom, B. R. (2014). Adolescents' aggression to parents: Longitudinal links with parents' physical aggression. The Journal of Adolescent Health, 55, 645-651. https://doi.org/10.1016/j.jadohealth.2014.05.008

Martínez, M. L., Estévez, E., Jiménez, T. I. y Velilla, C. (2015). Violencia filio-parental: Principales características, factores de riesgo y claves para la intervención [Child-Parent Violence: Main Characteristics, Risk Factors and Keys to Intervention. Papeles del Psicólogo, 36, 216-223. Recuperado de http://www.papelesdelpsicologo.es/pdf/2615.pdf

Miles, C. y Condry, R. (2016). Adolescent to parent violence: The police response to parents reporting violence from their children. Policing and Society, 26 ,

804-823. https://doi.org/10.1080/10439463.2014.989158

Pagani, L. S., Tremblay, R. E., Nagin, D., Zoccolillo, M., Vitaro, F. y McDuff, P. (2004). Risk factor models for adolescent verbal and physical aggression toward mothers. International Journal of Behavioral Development, 28, 528-537. https://doi.org/10.1080/01650250444000243

Pagani, L. S., Tremblay, R. E., Nagin, D., Zoccolillo, M., Vitaro, F. y McDuff, P. (2009). Risk factor models for adolescent verbal and physical aggression toward fathers. Journal of Family Violence, 24, 173182.

Pereira, R. (2006). Violencia filio-parental: Un fenómeno emergente [Parental Violence: An Emerging Phenomenon]. Mosaico, 36, 8-9.
Pereira, R. y Bertino, L. (2010). Los hijos que agreden a sus padres. La actitud del profesional de atención primaria [Children who attack their parents. The attitude of the primary care professional]. FMC. Formación Médica Continuada en Atención Primaria, 17(1), 39-47.

Pérez, T. y Pereira, R. (2006). Violencia filio-parental: Revisión de la bibliografía [Parental Violence: Review of Bibliography]. Mosaico, 36, 1-13.

Rico, E., Rosado, J. y Cantón-Cortés, D. (2017). Impulsiveness and child-to-parent violence: The role of aggressor's sex. The Spanish Journal of Psychology, 20. https://doi.org/10.1017/sjp.2017.15

Rodríguez, N. M., González-Álvarez, M. y García-Vera, M. P. (2011). Violencia de hijos a padres: La importancia de la exposición a la violencia interparental y de padres a hijos. Una revisión teórica [Violence of Children to Parents: The Importance of Exposure to Interparental Violence and from Parents to Children. A Theoretical Review. Psicopatología Clínica, Legal y Forense, 11, 123-141. Recuperado de: http://masterforense.com/pdf/2011/2011art7.pdf

Stewart, M., Burns, A. y Leonard, R. (2007). Dark side of the mothering role: Abuse of mothers by adolescent and adult children. Sex Roles, 56(3-4), 183-191. https://doi.org/10.1007/s11199-006-91482

Tew, J. y Nixon, J. (2010). Parent abuse: Opening up a discussion of a complex instance of family power relations. Social Policy and Society, 9, 579-589. https://doi.org/10.1017/S1474746410000291

Tibbitts, C. (1932). Reliability of factors used in predicting success or failure in parole. Journal of Criminal Law and Criminology, 22, 844-853. 


\title{
CHILD-TO-PARENT VIOLENCE RISK FACTORS: AN APPROACH WITH EXPERT JUDGEMENT
}

\author{
ISMAEL LOINAZ ${ }^{1}$, ANTONIO ANDRÉS-PUEYO ${ }^{1} \mathrm{Y}$ \\ ROBERTO PEREIRA ${ }^{2}$
}

\section{EXTENDED SUMMARY}

\section{Introduction}

Violence towards parents as well as elderly abuse are relatively new and little studied phenomena compared to intimate partner violence or sexual abuse. Youth violence has traditionally been studied in the context of general delinquency. It is even argued that the study of adolescent violence towards their parents has been underrepresented in public policies and criminological studies (Condry \& Miles, 2014). Although child-to-parent violence (CPV) sometimes shares characteristics with juvenile general violence and delinquency, it has different patterns and dynamics. One of the most used definitions of CPV is that of Cottrell (2001) which includes "Any behaviour that is deliberately harmful to the parent and used as a form of control [...]. The abuse may be physical, psychological (including verbal) or financial" (p. 3).

In recent years, the attention given to $\mathrm{CPV}$ in Spain has grown exponentially, surpassing that existing at the international context. In Spain, there are an average of 4600 official cases per year since 2007. However, a large number of cases do not have complaint. CPV still tends to be kept secret, often out of shame (Ghanizadeh \& Jafari, 2010; Kennedy, Edmonds, Dann, \& Burnett, 2010) and parents are reluctant to report until the situation is unsustainable. Spanish Youth Prosecutor's Office points out several peculiarities of CPV such as being rarely related to social exclusion situations, or that compared to other offenses, offenders are both male and female in proportions that tend increasingly to be equated (Fiscalía, 2013). Moreover, there is a need to communi- cate the risk level to the victim to adopt the appropriate protection measures.

\section{CPV risk factors}

The CPV literature has described a number of problematic variables both in offenders and in their families. As in other forms of violence, intergenerational transmission (Aroca, Bellver, \& Alba, 2012; Boxer, Gullan \& Mahoney, 2009) as well as the bidirectionality (offender being also the victim) (Ibabe \& Jaureguizar, 2011) have been used as explanatory factors. Violence between parents has been also related to a higher frequency of CPV (Boxer et al., 2009; Gámez-Guadix, \& Calvete, 2012). Other forms of victimization, as being bullied in the school, may also explain some aggressions (Cottrell \& Monk, 2004; Estévez, English, Emler, MartínezMonteagudo, \& Torregrosa, 2012; Loinaz \& Sánchez, 2015). The presence of other type of violence (to partners or relatives other than the parents) is characteristic of CPV judicial samples (Ibabe \& Jaureguizar, 2011).

An important set of risk factors are related to characteristics of the aggressor. The psychopathological symptomatology is frequent in young people who attack their parents (Calvete, Orue, \& Gámez-Guadix, 2013; Calvete, Orue, \& Sampedro, 2011; Castañeda, Garrido-Fernández, \& Lanzarote, 2012; Cuervo et al., 2008; GonzálezÁlvarez, Morán, \& García-Vera, 2011; Ibabe \& Jaureguizar, 2011; Ibabe, Arnoso, \& Elgorriaga, 2014a, 2014b; Kennedy et al., 2010). Compared to other juvenile offenders, CPV cases have lower empathy (Ibabe \& Jaureguizar, 2011) and lower self-esteem (Cuervo et al., 2008; González-Álvarez et al., 2011; Pereira \& Bertino, 2010). Another characteristic feature in these cases is the low frustration tolerance, high impulsivity and anger 
control difficulties (Calvete et al., 2011; Castañeda et al., 2012; Cuervo et al., 2008, Cuervo \& Rechea, 2010; González-Álvarez et al., 2011; Ibabe, Jaureguizar, \& Díaz, 2007). Finally, within these personality traits, narcissism or grandiosity ideas have also been linked to CPV (Calvete, 2008; Calvete et al., 2011).

Regarding the adaptation of the offenders, the most repeated factor is the academic difficulties (Cuervo et al., 2008, González-Álvarez, Gesteira, Fernández-Arias, \& García-Vera, 2010; González-Álvarez et al., 2011). On the other hand, antisocial behaviour and antisocial relationships have been associated with a greater number of risk factors (Cottrell \& Monk, 2004; Hong, Kral, Espelage, \& Allen-Meares, 2012; Ibabe et al., 2007).

Family issues are of special interest in CPV cases. A problematic educational style is often described (Aroca, Miró, \& Bellver, 2013, Castañeda et al., 2012, Cuervo et al., 2008, Cuervo \& Rechea, 2010). Related to this educational style, sometimes an inversion of the hierarchy may occur, and the offender supplants the parent's role of authority (Pérez \& Pereira, 2006; Stewart, Burns, \& Leonard, 2007). Other family risk factors are coercive cycles (Pagani et al., 2004, 2009), non-violent conflicts (Ibabe et al., 2007), and inability to establish coherent norms (Pereira \& Bertino, 2010). In addition, there are parental problems, such as addictions and other psychological disorders, which have been linked to CPV (Cuervo et al., 2008).

Along with risk factors, the literature describes protective factors that tend to be common in different types of violence and criminal forms. These factors have not been specifically described in CPV, but the family implication in therapy, future prosocial plans, social support, and family support can be considered protective.

Taking into account the literature review and the need of specific risk assessment tools for different types of violence and offenders, this project had the aim of proposing the first tool for CPV. A tool with the consensus of the professionals to whom it is intended is expected.

\section{Method}

\section{Participants}

A total of 160 professionals participated in a survey, and 112 complete answers were analysed. Respondents included psychologist $(39.3 \%)$, social educators $(24.1 \%)$, police officers $(9.8 \%)$, social workers $(6.3 \%)$ and university researchers $(5.4 \%)$ among others. The mean age was 41 years $(d t=9.9$, range $=23-66)$ and $46.4 \%$ were women. Most of them (58\%) had training in violence risk assessment, $67.6 \%$ of them academic (studies such as criminology or forensic psychology) and $80 \%$ in specific workshops. Also, the majority of respondents $(79.5 \%)$ were working directly on CPV cases. Of those who did not currently work in the field, $43.4 \%$ had done so previously.

\section{Survey}

A survey was developed through a review of the CPV literature, containing 28 factors (Table 1), grouped into four dimensions: 1) characteristics of violence; 2) characteristics of the offender; 3) family characteristics; and 4) protective factors. Respondents were asked with closed-ended questions about the adequacy of these factors to assess the risk of CPV (adequate, don't know, inadequate), as well as opinion regarding the proposal of a tool to assess risk in these cases (Table 2).

\section{Procedure}

Several professionals were contacted by email to request their collaboration. This contact included universities, treatment centres, juvenile justice services, and police forces. In addition, the survey was available on the websites of the Spanish Society for the Study of Childto-Parent Violence (SEVIFIP), Euskarri (Centre for training and intervention in child-to-parent violence) and the group Deusto Stress Research. Participation took place between April and June 2014. After analysing the results of the survey, the first version of the tool was developed and seven pilot applications were carried out at the 
Euskarri centre (Bilbao) in June 2014. The objective of this phase was to test the tool. In December 2014, a clinical session was also held with a panel of experts at the Amalgama 7 centre (Barcelona).

\section{Results}

\section{Adequacy of risk factors}

The professionals' opinion regarding the adequacy of the risk factors for the assessment of CPV cases is presented in Table 1. The group of variables considered more adequate (percentage higher than $90 \%$ ) corresponded to family issues (violence among parents, cohabiting problems, educational style, family support and family support in therapy). Regarding the offender, the most appropriate variables were affective style, substance abuse and anger management problems. Narcissism or ideas of grandiosity $(67.3 \%)$, non-violent conflicts between parents $(66.4 \%)$, single parenthood $(62.8 \%)$ and the adoptive family (64\%) received less professional support.

\section{Opinion about the tool proposal}

The proposal was considered quite $(45.65 \%)$ or very (38.04\%) necessary, that covered quite a lot $(70.65 \%)$ or perfectly $(10.87 \%)$ professional needs, that was quite $(54.35 \%)$ or very useful $(14.13 \%)$ for the work in cases of CPV, that covered the areas of interest $(88 \%)$, and that it could be simple to use $(89.13 \%)$. Regarding the utility, respondents considered it: quite $(48.91 \%)$ or very useful $(6.52 \%)$ to assess the possible evolution of the case; quite $(65.2 \%)$ or very $(20.65 \%)$ useful for assessing intervention needs; quite $(56.04 \%)$ or very $(14.29 \%)$ useful to recommend a treatment; quite $(37.36 \%)$ or very $(10.99 \%)$ useful for predicting recidivism; and quite $(47.83 \%)$ or very $(17.39 \%)$ useful for assessing family risk.

Table 2 compares these opinions between those who worked or not in CPV, and those who did or did not have training in violence risk assessment. The only differences were that those who did not work in CPV considered more necessary the tool and more useful to predict recidivism, and those who were not trained in violence risk assessment considered it more useful to assess family risk.

\section{Proposals of the respondents}

Respondents did different improvement proposals, like new risk factors that they considered relevant and were not present. Nine new factors were included: age at onset of CPV; commission of CPV by siblings; attitudes towards the use of violence; lack of values; type of violence; failure in previous interventions; migration, family reunification, temporary separation between parents and children, culture of origin; therapeutic alliance; victims of gender violence (the mother or the abusive daughter).

Following the pilot applications and discussion groups, several modifications were included that led to version 2 of the tool. The items that had more empirical support remained as main factors. The others (e.g., single parenthood, adoptive family, and mother victim), became part of the socio-demographic variables. The risk factor 7 (see table 1) was divided into two in the tool draft (7. empathy problems, and 8. self-esteem problems). Violent attitudes, failure in previous interventions, motivation for change, and therapeutic alliance were incorporated as new factors. The final version of the tool (v2.0, available on request to the authors) contains a total of 24 risk factors, 6 protective factors and 15 case identification variables (including sociodemographic and other risk factors).

\section{Discussion}

The literature review reveals some variables that are repeated in the research on CPV and that can be considered risk factors. These variables were submitted in 2014 to professionals to consider their relevance to be incorporated into a CPV risk assessment tool. Many of the factors had been reviewed previously (Ibabe, Jauregizar, \& Bentler, 2013, Lozano, Estévez, \& Carballo, 2013), and also after this survey (Martínez, Estévez, Jiménez, \& Velilla, 2015). Others, however, are less common and not 
included in these reviews. The research has also pointed out the need to agree on definitions and develop tools for assessing the risk of violence in cases of CPV (Holt, 2013), as well as to understand how certain variables become risk factors in specific populations (Holt, 2012).

The survey findings were used to elaborate a tool with the agreement of the professionals who work in their daily practice with CPV cases and are intended to be the final users, something considered crucial since the origins of the development of crime prediction tools (Tibbitts, 1932) and part of the process of creating and reviewing the main tools of structured professional judgment (see Douglas et al., 2014). Regarding the tool, the main conclusion is that it was considered necessary and useful. Risk factors were supported and nine proposals of less common factors were included (e.g., single parenthood, adoptive family, death of a parent, aggressor siblings, age at onset of CPV). The pilot application of the first version allowed to solve the main problems related to its use in practice. After this, it was proposed a tool (available on request to authors) that is currently being applied experimentally in different contexts.

The work still represents a first phase in the development of a risk assessment tool for CPV. The following steps should establish its final content and its properties (e.g., predictive ability, convergent validity, reliability), including its differential contribution compared to other available tools such as SAVRY (Borum, Bartel, \& Froth, 2006), for example, for juvenile samples (although we must remember that those involved in CPV are not necessarily minors). 